\title{
A role for the tetraspanin CD81 on the late steps of HIV-1 replication in T-lymphoblastic cells
}

\author{
Delphine Muriaux
}

From $16^{\text {th }}$ International Symposium on HIV and Emerging Infectious Diseases

Marseille, France. 24-26 March 2010

\section{Background}

HIV-1 uses cellular co-factors for virion formation and release, and is able to incorporate host cellular proteins in the viral particles, such as tetraspanins which serve as gateways for HIV-1 egress. Here, we investigated the implication of several tetraspanins on HIV-1 formation and release in chronically infected T-lymphoblastic cells, a model that permits the study of the late steps of HIV1 replication in persistent infected cells.

\section{Methods}

HIV-1 infected MOLT cells were analyzed for HIV-1 production by RT assays and Western blot analysis. Gag-Tetrapanins associations were analyzed by immunoprecipitations in the purified virions and in the infected cells and by immunofluorescence confocal microscopy analysis. Down-regulation of CD81 expression in HIV-1 chronically infected MOLT cells was performed by shRNA lentiviral vectors and infectivity was monitored on SupT1 cells.

\section{Results}

Our data revealed that HIV-1 Gag and Env structural proteins colocalized with specific tetraspanins in the form of clusters at the cell surface. Co-immunoprecipitation experiments showed that viral Gag proteins interact, directly or indirectly, with the CD81 tetraspanin, and less with CD82, but not with Lamp2 or CD45, in tetraspaninenriched microdomains composed of CD81/CD82/CD63.

When HIV-1 producing cells were treated with antiCD81 antibodies or upon CD81 silencing by RNA interference, HIV-1 release was significantly impaired and its infectivity on SupT1 lymphocytes was modulated. We observe that CD81 downregulation in HIV-1 infected
T-lymphoblastic cells resulted in Gag redistribution at the cell surface and an increase in infectivity.

\section{Discussion}

Our results highlight a critical role for CD81 on HIV assembly in T lymphoblastic cells [1], which was also reported in HIV-1 infected monocytes derived macrophages [2], and on HIV transmission in CD4 + T cells [3] and dendritic cells [4]. In addition, our findings extend the notion that even if HIV-1 assembly can occur on tetraspanin-enriched microdomains containing CD81, the incorporation of CD81 in the viral particles restrict HIV-1 infectivity. This notion can be extend to other cell membrane proteins, such as Hdlg, a cell-cell junction protein, that can also modulate HIV-1 infectivity [5].

Published: 11 May 2010

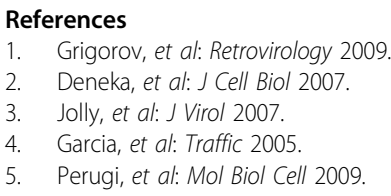

doi:10.1186/1742-4690-7-S1-P6

Cite this article as: Muriaux: A role for the tetraspanin CD81 on the late steps of HIV-1 replication in T-lymphoblastic cells. Retrovirology 20107 (Suppl 1):P6. 